\title{
Efecto de la distribución de hidrocarburos SARA sobre las propiedades de residuales de vacío
}

\author{
Effect of the Distribution of Hydrocarbons SARA \\ Over the Properties of Vacuum Residual
}

\author{
Meza-Ávila Alejandra \\ Universidad Central de Venezuela, Venezuela \\ Correo:alejamezavila@hotmail.com \\ Da Fonseca-Rodríguez Andreina \\ Universidad Central de Venezuela, Venezuela \\ Correo:andreina_908@hotmail.com \\ Ruiz-Hernández Ernesto \\ Universidad Central de Venezuela, Venezuela \\ Correo:ernestoruiz22@hotmail.com
}

\author{
Pérez-Santodomingo Narciso \\ Universidad Simón Bolivar, Venezuela \\ Correo:naperez@usb.ve \\ Rincón-Polo Gladys \\ Ateneo-Senescyt, Escuela Superior Politéenica del Litoral \\ FIMCBOR-ESPOL, Ecuador \\ Correo:grincon@usb.ve
}

Información del artículo: recibido: febrero de 2015, aceptado: junio de 2016

\section{Resumen}

Con la finalidad de evaluar el efecto de la composición SARA de residuales de vacío sobre sus propiedades físicoquímicas más importantes, se realizó la separación de tres residuales de vacío de origen venezolano en sus 4 grupos fundamentales: Saturados, Aromáticos, Resinas y Asfaltenos (SARA), preparando a partir de ellos cuatro nuevos residuales, denominados residuales base, con concentraciones másicas de al menos 50\% de cada uno de estos grupos, respectivamente. Estos residuales base se caracterizaron a partir de la relación $\mathrm{C} / \mathrm{H}$, análisis inmediato, concentraciones de metales pesados y carbón Conradson, encontrándose que para aquellos que contenían altas concentraciones de los grupos saturados y aromáticos, la relación $\mathrm{C} / \mathrm{H}$ era menor, la proporción de material volátil era mayor y la concentración de metales pesados y de carbón Conradson eran menores que en el caso de los residuales con mayores concentraciones de grupos resínicos y asfalténicos. Se obtuvo además que la posibilidad de craqueo y formación de coque del residual está directamente relacionada con la presencia de estos dos últimos grupos de hidrocarburos en el crudo.

\section{Descriptores:}

- SARA

- coquización retardada

- líquidos condensables

- coque 


\begin{abstract}
In order to evaluate the effect of the SARA composition of vacuum residuum on its major physicochemical properties, the separation of three vacuum residuum of Venezuelan origin was done in its four main groups: Saturates, Aromatics, Resins and Asphaltenes (SARA), preparing from these four new residuum, called base residuals, with mass concentrations of each of these groups at least $50 \%$, respectively. These base residuals were characterized in based on the $\mathrm{C} / \mathrm{H}$ relation, immediate analysis, concentrations of heavy metals and Conradson Carbon, being that for those containing high concentrations of saturated and aromatic groups, $\mathrm{C} / \mathrm{H}$ relation was lower, volatile material was greater, and the concentration of heavy metals and Conradson carbon were lower than in the case of residuum with higher concentrations of resin and asphaltene groups. Also it was obtained that the possibility of cracking and coke formation of the residuum, is directly related to the presence of the latter two groups in the crude oil.
\end{abstract}

\section{Introducción}

Venezuela cuenta con una enorme reserva en crudos (PDVSA, 2013), que corresponde en su mayoría a crudos pesados y extrapesados. Con la finalidad de obtener el mayor beneficio en este tipo de crudos, es necesario mejorar su calidad por medio de procesos de conversión profunda como los de coquización retardada, flexicoquización y coquización fluidizada. De estos procesos, el más utilizado en Venezuela es la coquización retardada, que consiste en un tratamiento fisicoquímico térmico en donde ocurren fundamentalmente dos tipos de reacciones endotérmicas; una de craqueo térmico y otra de polimerización-condensación (Linares, 2003; López, 2004).

La química detallada de este proceso se desconoce, debido a que implica una serie de reacciones complejas. Como un aporte para la solución a esta problemática se propone evaluar la posibilidad de generar residuales de vacío de composición controlada que posteriormente se utilicen en la evaluación del efecto del tipo de alimentación sobre el rendimiento y calidad de los productos de la coquización retardada.

Para ello, se procesaron tres residuales de vacío de origen venezolano identificados según su origen como: Petrozuata, Amuay y Cardón, por medio de tratamientos físicos y el ajuste de la cantidad presente en ellos de cada uno de los cuatro grupos característicos de hidrocarburos: SARA (Saturados, Aromáticos, Resinas y Asfaltenos), para obtener cuatro nuevos residuales denominados: "residuales bases" (cada uno con un criterio para su preparación, la cual debe contener una composición mayor a $50 \%$ en peso de una de las fracciones SARA) que puedan emplearse en investigaciones futuras de coquización retardada a escala laboratorio, con el fin de establecer relaciones entre el tipo de alimentación y los productos obtenidos, por medio de la caracterización fisicoquímica tanto de la alimentación como de los productos.

\section{Metodología}

La metodología se divide en dos partes: a) preparación de los residuales base y b) caracterización de los residuales base.

\section{a) Preparación del residual base}

A tres residuales de vacío de origen venezolano nombrados por sus denominaciones comerciales: Amuay, Cardón y Petrozuata, se les determinó la composición de cada fracción de hidrocarburos a través del Análisis SARA siguiendo la Norma ASTM D 4124-09 (ASTM International, 2009). El residual base es el residual de vacío con composición controlada que alimenta al proceso de coquización retardada a escala laboratorio.

A partir de los residuales comerciales se prepararon concentrados de la fracción de hidrocarburo, que se logró por medio de una variación de la norma ASTM D4124-09 (ASTM International, 2009) desarrollada y probada exitosamente por López (2004). El método empleado se basa en el mismo principio de adsorción y afinidad con los solventes utilizado en la norma ASTM D4124-09 (ASTM International, 2009), pero en vez de operar en columna, se emplean varios balones, entre los cuales se distribuye equitativamente una cantidad de 25 gramos de alúmina por gramo de maltenos. La diferencia entre esta variación y el método original, es que la variación permite obtener un mayor volumen del residual, por lo que a este método se le denominó "Macro-SARA". 
Como requisito se exigió que los residuales base contengan al menos $50 \%$ en peso de una de las fracciones de hidrocarburo: Saturados, Aromáticos, Resinas o Asfaltenos. Cada uno de los cuatro tipos de residuales base (uno por cada tipo de fracción de hidrocarburo) se prepararon mezclando el residual comercial con un concentrado de la fracción de hidrocarburo preparado previamente (Saturados, Aromáticos, Resinas o Asfaltenos) obtenida a partir del mismo residual comercial. Se agrega la cantidad necesaria del concentrado de la fracción de hidrocarburo hasta garantizar que la concentración en peso de dicha fracción en el residual base sea al menos de $50 \%$ en peso. Con este requisito se buscaba que el comportamiento del residual preparado se acercara lo más posible al de la fracción mayoritaria. No se trabajó con porcentajes mayores a este, debido a limitaciones en el proceso de separación, sobre todo en el caso de la fracción de Saturados que posee una cantidad máxima de $7 \%$ p/p en los residuales base.

Para garantizar la homogeneidad del residual preparado, se sometió el residual base a un proceso de calentamiento-agitación donde se le incorporó lentamente el concentrado correspondiente de la fracción preparada. Este proceso se realizó hasta garantizar que la mezcla preparada fuera homogénea.

\section{b) Caracterización de los residuales base}

Todos los residuales de vacío que se trabajaron en esta investigación se analizaron por medio de los siguientes ensayos:

1) Contenido de carbón Conradson: según la Norma ASTM D189-06 (ASTM International, 2014).

2) Análisis inmediato, mediante analizador termogravimétrico TAQ-500 haciendo uso de la norma ASTM D5142 (ASTM International, 2010): este análisis comprende la determinación de humedad, material volátil y cenizas.

3) Análisis elemental: esta prueba determina el contenido de carbono e hidrógeno presente en el residual. Se realiza bajo la Norma COVENIN 2596-89 (Comisión Venezolana de Normas industriales, 1989) (relación $\mathrm{C} / \mathrm{H}$ ), y la determinación del contenido de azufre total se realizó bajo la Norma ASTM D4239 (ASTM International, 1998).

4) Contenido de metales: la determinación del contenido de metales ( $\mathrm{V}$ y Ni) se realizó según la Norma EPA6010 (United States Enviromental Protection Agency, 2007). El método describe la determinación de multi-elementales por ICP-AES utilizando sistemas ópticos secuenciales o simultáneos y visualización axial o radial del plasma.

5) Termogravimetría: se opera bajo la Norma ASTM E1641 (ASTM International, 2004). El equipo utilizado es una balanza marca TA Instrument, modelo TAQ500, la cual opera bajo una temperatura máxima de $1000^{\circ} \mathrm{C}$.

\section{Resultados y discusión de resultados}

a) Caracterización de los residuales de vacío venezolanos

Al hacer la caracterización SARA de cada uno de los residuales de vacío venezolanos se obtiene la figura 1 , donde se representa la cantidad en peso que contenía cada uno de los residuales preparados de las fracciones SARA. En ella se observa que ninguno de los residuales contenía más de $50 \%$ en peso de una de las fracciones de hidrocarburos, por lo que el residual debió mezclarse con concentrados de las diferentes fracciones de hidrocarburo para la obtención de los cuatro tipos de residuales base.

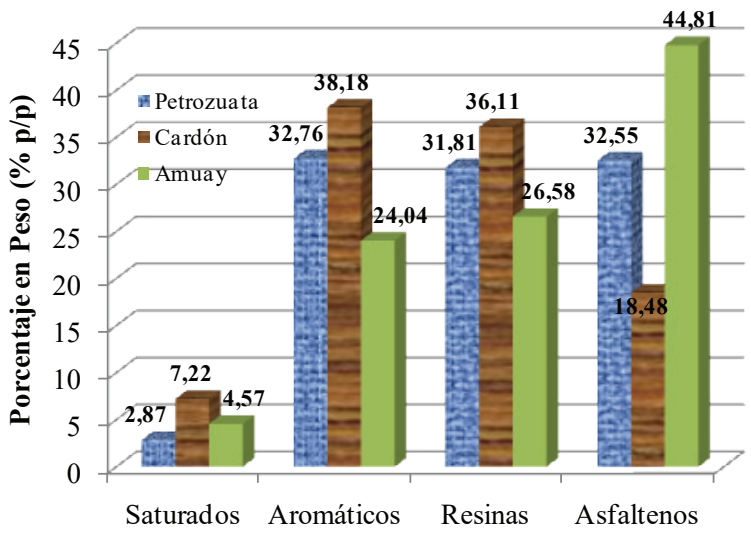

Figura 1. Composición SARA de los residuales disponibles

En la tabla 1 se indican los residuales venezolanos utilizados para preparar cada uno de los residuales base indicando el tipo de concentrados de fracciones de hidrocarburos que se usa con cada residual venezolano.

Los resultados del análisis SARA de los residuales base preparados se muestran en la tabla 2. En ella se resalta el contenido total de la fracción de hidrocarburo que lo hace rico en una fracción específica. 
DOI: https://doi.org/10.1016/j.riit.2016.11.003

Efecto de la distribución de hidrocarburos SARA sobre las propiedades de residuales de vacío

Tabla 1. Residuales venezolanos utilizados para la obtención de los residuales preparados

\begin{tabular}{|c|c|c|c|c|}
\hline \multirow{2}{*}{ Tipo de residuales base } & \multirow{2}{*}{ Identificación de residuales base } & \multicolumn{3}{|c|}{ Residual Comerciales } \\
\hline & & Amuay & Cardón & Petrozuata \\
\hline $\begin{array}{l}\text { Residual rico en hidrocarburos } \\
\text { saturados }\end{array}$ & R2 & - & - & $X$ \\
\hline $\begin{array}{l}\text { Residual rico en hidrocarburos } \\
\text { Aromáticos }\end{array}$ & $\mathrm{R} 4$ & - & - & $X$ \\
\hline $\begin{array}{l}\text { Residual rico en hidrocarburos } \\
\text { resinas }\end{array}$ & R5 & - & $X$ & - \\
\hline $\begin{array}{l}\text { Residual rico en hidrocarburos } \\
\text { asfaltenos }\end{array}$ & R6 & $X$ & - & - \\
\hline
\end{tabular}

Tabla 2. Composición SARA de los residuales base preparados

\begin{tabular}{ccccc}
\hline $\begin{array}{c}\text { Composición } \\
\text { residual }\end{array}$ & \multicolumn{4}{c}{ Concentración $(\% \mathrm{p} / \mathrm{p})$} \\
\hline Residual & $\begin{array}{c}\text { Saturados } \\
(\%)\end{array}$ & $\begin{array}{c}\text { Aromáticos } \\
(\%)\end{array}$ & $\begin{array}{c}\text { Resinas } \\
(\%)\end{array}$ & $\begin{array}{c}\text { Asfaltenos } \\
(\%)\end{array}$ \\
R2 & 56.58 & 14.65 & 14.22 & 14.55 \\
R4 & 1.99 & 53.45 & 22.02 & 22.53 \\
R5 & 5.21 & 27.54 & 53.92 & 13.33 \\
R6 & 4.11 & 21.60 & 23.88 & 50.42 \\
\hline
\end{tabular}

b) Caracterización de los residuales preparados (residuales bases)

Los resultados de la caracterización de los residuales base y de los residuales comerciales a través de las diferentes pruebas de laboratorio que se indican en la primera columna se resumen en la tabla 3. En esta tabla también se incorpora la caracterización del residual mesa debido a que este se utiliza como residual de referencia (Salazar, 2012).

1) Carbón Conradson: El resultado de contenido de Carbón Conradson muestra la tendencia que tiene cada tipo de residuales sobre la producción de coque, por lo que se espera que el residual base R6 al tener la mayor proporción de asfaltenos tenga el mayor valor del grupo. Por el contrario, el residual base R2 cuya proporción de parafinas es la más alta de todos los residuales base, solo está presente con un poco más $10 \%$. Comparando el porcentaje de Carbón Conradson obtenido para los residuales base, respecto al de los residuales comerciales, se tiene que al aumentar la concentración de una de la fracciones de hidrocarburos se modifica el contenido de carbón fijo. Se observa que para R2 y R4 el porcentaje de Carbón Conradson tiende a disminuir, mientras que para R5 y R6 ocurre lo contrario. Este comportamiento se debe a que tanto la fracción de resinas como la de asfaltenos, se constituye por moléculas pesadas que se favorecen por la formación de coque (Requena, 2007). En el caso de las fracciones de saturados y aromáticos, estas son moléculas más livianas que se favo-

recen por la producción de líquidos, que provoca una disminución en la formación de coque (Linares, 2003).

2) Análisis inmediato: Existe una disminución del contenido de material volátil a medida que el grupo mayoritario del residual se hace más pesado, esto se debe a que la relación $\mathrm{C} / \mathrm{H}$ va en aumento (Requena, 2007). Respecto a los residuales R4, R5 y R6 se tiene que el porcentaje de material volátil se redujo en menor proporción en comparación con el primer residual (R2), por lo que se presume que al aumentar la concentración de la fracción pesada se realiza una reestructuración o rearreglo de las moléculas. En el caso del contenido de humedad, se encuentra que la humedad en los residuales disminuye, lo cual se asocia al hecho de que el origen de la alimentación corresponde a residuales de vacío cuyo contenido de agua se extrae de los procesos anteriores.

3) Relación C/H y contenido de Azufre: A medida que el componente mayoritario del residual es más pesado, la relación $\mathrm{C} / \mathrm{H}$ es mayor. Este comportamiento indica que al incrementar las moléculas de mayor peso aumenta el grado de condensación de los componentes del residual (Requena, 2007). Por lo que el residual R6 correspondiente a asfaltenos, presenta la mayor relación $\mathrm{C} / \mathrm{H}$ y por lo tanto, posee un mayor rendimiento en la producción de coque. En cambio, para el residual R2 (residual saturado), la relación C/H es menor a uno (1) por lo que presenta un mayor rendimiento en la producción de gases y livianos. Comparando la relación $\mathrm{C} / \mathrm{H}$ de los residuales 
base con la de los residuales comerciales, se tiene que para $\mathrm{R} 2$ la relación $\mathrm{C} / \mathrm{H}$ es mayor en el residual base. $\mathrm{Al}$ aumentar la presencia de saturados se esperaría que el contenido de hidrógeno aumentara, por lo que se presume que esta diferencia se debe al efecto que pueden tener la presencia de los otros grupos de hidrocarburos en la mezcla del residual para la alimentación del coquizador preparado sobre esta propiedad.

Respecto a los residuales base R4, R5 y R6 se observa un aumento en la relación $\mathrm{C} / \mathrm{H}$ a medida que se aumenta la concentración de moléculas cada vez más pesadas, se aumenta el contenido de carbono disminuyendo el de hidrógeno. En la tabla 3 también se incluye la relación $\mathrm{C} / \mathrm{H}$ del residual mesa analizado por (Salazar, 2012), valor que constata la reproducibilidad de los resultados obtenidos.

El contenido de azufre aumenta en la medida que incrementa la concentración en las fracciones más polares y de mayor peso, tal como se observa en R5 y R6. Esto ocurre como consecuencia de la presencia de una mayor cantidad de heteroátomos de azufre asociada la estructura del hidrocarburo (Salazar, 2012).

4) Contenido de metales: La mayor proporción de níquel y vanadio se concentra en la fracción más polar o más pesada del crudo, lo que es un indicativo de que la mayor parte de estos elementos se encuentran en forma de compuestos porfirínicos, no porfirínicos y sales de ácidos nafténicos (Campos, 2012).

Comparando los valores obtenidos con el contenido de metales de los residuales de vacío con el residuo de Petrozuata, se observa que las modificaciones en la concentración de metales se relaciona con el contenido de las fracciones de hidrocarburo en la alimentación: al incrementar la presencia de fracciones livianas (saturados y aromáticos) el contenido total de metales se encuentra por debajo del residual comercial, mientras que al aumentar la presencia de fracciones pesadas (resina y asfaltenos) el contenido total de metales se incrementa.

En la tabla 3 se muestra también el contenido total de metales (vanadio + níquel) en los residuales bases y los comerciales. En esta fila de la tabla 3, se observa que incrementos en la concentración de parafinas y aromáticos en los residuales bases R2 y R4, conllevan una disminución en el contenido de metales. Esto se debe a que los saturados no tienen estos metales asociados en su estructura. Respecto a los aromáticos, estos solo asocian la presencia de heteroátomos de azufre (Requena, 2007).

Para el residual base $\mathrm{R} 5$ (residual resínico) no se presentan cambios, por lo que no se asocia esta fracción con la presencia de altos contenidos de metales. Por último, se tiene que para R6 (correspondiente al residual asfalténico) tal como se esperaba, el contenido de metales aumenta significativamente.

5) Termogravimetría: El termograma de los residuales preparados (figura 2) muestra la relación que tiene la masa residual con la temperatura. Para los residuales base R2, R4, R5 y R6 se tiene que el porcentaje de masa residual (descomposición) es similar, por lo que estos cuatro residuales tienen una composición muy parecida en compuestos con temperatura de descomposición menor de $500^{\circ} \mathrm{C}$.

A partir de aproximadamente $500^{\circ} \mathrm{C}$ la masa residual es prácticamente constante. Este resultado es significativo desde el punto de vista de la coquización retardada, debido a que indica que la temperatura mínima para llevar a cabo el proceso de coquización debe ser $500^{\circ} \mathrm{C}$, punto en el cual se han craqueado la mayoría de los compuestos. Por lo tanto, a partir de esta tempe-

Tabla 3. Caracterización de los residuales de vacío empleados en la investigación

\begin{tabular}{|c|c|c|c|c|c|c|c|c|}
\hline $\begin{array}{c}\text { Prueba de } \\
\text { laboratorio }\end{array}$ & $\mathrm{R} 2$ & R4 & R5 & R6 & Petrozuata & Amuay & Cardón & $\begin{array}{l}\text { Residual } \\
\text { Mesa* }^{*}\end{array}$ \\
\hline $\begin{array}{l}\text { Carbón } \\
\text { Conradson (\%) }\end{array}$ & 11.82 & 22.16 & 24.13 & 33.98 & 24.20 & 20.70 & 22.60 & - \\
\hline Humedad (\%) & 0.09259 & 0.22260 & 0.48080 & 0.01082 & - & - & - & - \\
\hline $\begin{array}{l}\text { Material volátil } \\
(\%)\end{array}$ & 93.00 & 86.31 & 84.11 & 82.74 & - & - & - & - \\
\hline Relación C/H & 0.809 & 1.155 & 1.354 & 1.690 & 0.658 & 0.691 & 0.812 & 0.620 \\
\hline Azufre $(\% \mathrm{p} / \mathrm{p})$ & 3.66 & 4.67 & 4.58 & 4.69 & 5.53 & 3.93 & 4.42 & - \\
\hline Níquel (ppm) & 92 & 103 & 112 & 120 & 133 & 65 & 93 & - \\
\hline Vanadio (ppm) & 343 & 548 & 780 & 984 & 659 & 617 & 799 & - \\
\hline $\begin{array}{l}\text { Níquel + Vanadio } \\
\text { (ppm) }\end{array}$ & 435 & 651 & 892 & 1104 & 792 & 682 & 892 & - \\
\hline
\end{tabular}

* Residual referencia reportado por (Salazar, 2012) 
ratura $\left(500^{\circ} \mathrm{C}\right)$ no se observarían efectos significativos en cuanto al rendimiento de los productos de mayor valor comercial.

Desde el punto de vista cinético se tiene que el residual (R) se transforma en Gases y Vapores (G) al mismo tiempo que en Sólidos (S). Asumiendo que las reacciones de la coquización retardada son independientes entre sí, se plantean las siguientes ecuaciones químicas basadas en las reacciones presentadas por (Perea, 2009)

$R^{\Delta, k 1} G$

$R^{\Delta, k 2} S$

Cada reacción con su respectiva constante de rapidez $\left(\mathrm{k}_{1} \mathrm{y}_{2}\right)$.

En el análisis termogravimétrico se puede observar que a una temperatura entre $150-500^{\circ} \mathrm{C}$ se favorece la primera reacción (Ec. 1) que consiste en las reacciones de craqueo, ya que en este intervalo de temperatura en la figura 2 se observa una gran pérdida de masa correspondiente a la producción de volátiles. Luego a temperaturas mayores de $500^{\circ} \mathrm{C}$ se favorece la segunda reacción (Ec. 2) por lo que se tiene que a temperaturas mayores de $500^{\circ} \mathrm{C}$ predominan las reacciones de condensación, así, $\mathrm{k}_{3}>\mathrm{k}_{2}$, es decir, se llevan a cabo las reacciones de craqueo, pero se tienen rendimientos muy bajos debido a que la pérdida de masa es baja entre 500 y $900^{\circ} \mathrm{C}$, como se observa en la figura 2 .

Adicionalmente, al comparar las cuatro curvas, se tiene que a más de $600^{\circ} \mathrm{C}$ las curvas forman escalones, observando que el residual con mayor pérdida de masa es R2, ya que posee una mayor concentración de livianos (saturados), luego le siguen R4, R5 y R6. Este resultado se debe a que el grupo funcional mayoritario influye en el punto de ebullición del residual, es decir, a medida que el residual se hace más condensado, aumenta el punto de ebullición (Favenec, 1998).

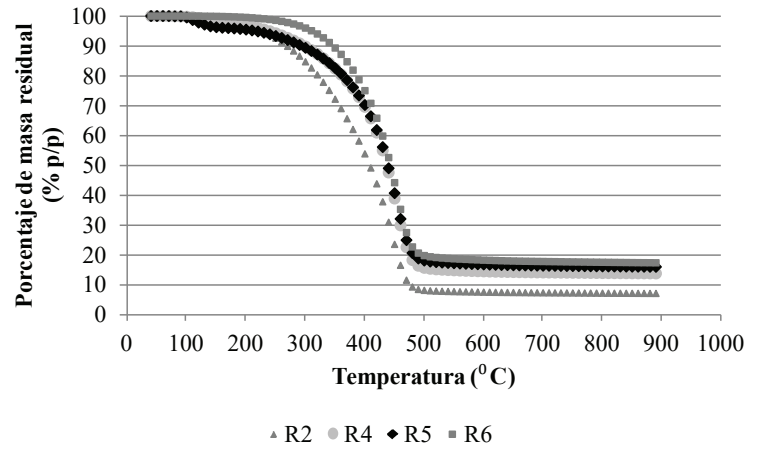

Figura 2. Análisis termogravimétrico de los residuales preparados

\section{Conclusiones}

Fue posible generar residuales de vacío de composición SARA controlada a partir de tratamientos físicos de residuales de origen venezolano. Los residuales obtenidos mostraron que sus propiedades fisicoquímicas son dependientes del tipo de grupo de hidrocarburo SARA que los conforman, dando como resultado que en la medida que el residual esté conformado por grupos saturados y aromáticos, las propiedades obtenidas del análisis del residual indican que este tendrá una menor probabilidad de producir coques en un proceso de coquización retardada, lo contrario sucede cuando el residual está fundamentalmente conformado por resinas y asfaltenos.

Se encontró que el análisis termogravimétrico permite observar el comportamiento cinético de los residuales durante el proceso de coquización retardada. Este análisis permitió verificar que la temperatura mínima para llevar a cabo el proceso de coquización retardada de forma satisfactoria es $500^{\circ} \mathrm{C}$ y que a temperaturas menores a esta, se favorecen las reacciones de craqueo térmico, mientras que a temperaturas mayores se favorecen las reacciones de condensación. Adicionalmente, a través del análisis termogravimétrico para los cuatro residuales preparados, se demostró la dependencia de la posibilidad de craqueo o polimerización con el tipo de grupo de hidrocarburo SARA, donde la posibilidad de craqueo es inversamente proporcional a la de polimerización, con el máximo valor. Además corresponde al grupo de saturados, seguido tanto de los aromáticos como de las resinas con magnitudes semejantes, quedando por último el grupo de asfaltenos con la menor posibilidad de craqueo.

\section{Referencias}

ASTM International. ASTM D4239. Standard test method for sulfur in the analysis sample of coal and coke using high-temperature tube furnace combustion, 1998.

ASTM International. ASTM E1641. Standard test method for decomposition kinetics by Thermogravimetry, 2004.

ASTM International. ASTM D4124-09. Standard test method for separation of asphalt into four fractions, 2009.

ASTM International. ASTM D5142. Standard test methods for proximate analysis of the analysis sample of coal and coke by instrumental procedures, 2010.

ASTM International. ASTM D189-06. Standard test method for Conradson Carbon residue of petroleum products, 2014.

Campos G. Estudio de recuperación de vanadio y niquel en un crudo pesado y en su fracción de asfaltenos y maltenos por ICP-OES. Ciencia, volumen 20 (número 1): enero-marzo 2012: $52-59$. 
DOI: https://doi.org/10.1016/j.riit.2016.11.003

Meza-Ávila Alejandra, Da Fonseca-Rodríguez Andreina, Ruiz-Hernández Ernesto, Pérez-Santodomingo Narciso, Rincón-Polo Gladys

Comisión Venezolana de Normas Industriales. Norma COVENIN 2596-89. Carbón y coque. Determinación del contenido de carbono e hidrógeno, 1989.

Favennec J. Petroleum Refining: Refining and Operation Management, volumen 5, Editions Technip, París, 1998.

Linares A. Estudio de la naturaleza química de la carga al coquificador retardado y su relación con el lecho de coque formado en el tambor (trabajo de grado, ingeniería química), Universidad Central de Venezuela, Caracas, 2003.

López M. Estudio de las reacciones de condensación/polimerización de las fracciones SARA de un residual de refinación (trabajo de grado, licenciatura en química), Universidad Simón Bolívar, Sartenejas, 2004.

PDVSA 2013 [en línea]. [Fecha de consulta: 21 de enero de 2013]. Disponible en: http://www.pdvsa.com/PESP/Pages_pesp/aspectostecnicos/produccion/reservas.html

Perea Y.Y. Implementación de una unidad de delayed coking como mejora de los rendimientos y optimización del fraccionamiento de crudo pesado (tesis), Universidad Nacional de Ingeniería, Lima, Perú, 2009.

Requena A. Formación de mesofase en crudos pesados y sus residuales, Trabajo de Ascenso, Universidad Simón Bolívar, Sartenejas, 2007.
Salazar S. Estudio del proceso de coquización retardada a escala de laboratorio (trabajo de grado, maestría en química), Universidad Simón Bolívar, Sartenejas, 2012.

United States Environmental Protection Agency. EPA-6010. Inductively coupled plasma-atomic emission spectrometry, 2007.

\section{Este artículo se cita: \\ Citación estilo Chicago}

Meza-Ávila, Alejandra, Andreina Da Fonseca-Rodríguez, Ernesto Ruiz-Hernández, Narciso Pérez-Santodomingo, Gladys RincónPolo. Efecto de la distribución de hidrocarburos SARA sobre las propiedades de residuales de vacío. Ingeniería Investigación y Tecnología, XVII, 04 (2016): 437-443.

\section{Citación estilo ISO 690}

Meza-Ávila A., Da Fonseca-Rodríguez A., Ruiz-Hernández E., Pérez-Santodomingo N., Rincón-Polo G. Efecto de la distribución de hidrocarburos SARA sobre las propiedades de residuales de vacío. Ingeniería Investigación y Tecnología, volumen XVII (número 4), octubre-diciembre 2016: 437-443.

\section{Semblanzas de los autores}

Meza-Ávila Alejandra. Candidata a doctora en ingeniería por la Universidad Simón Bolívar. Actualmente es MSc. en ingeniería química por la Universidad Central de Venezuela, 2009 e ingeniera química por la Universidad Central de Venezuela, 2001. Es profesor agregado adscrito al Departamento de Termodinámica y Fenómenos de Transporte, Escuela de Ingeniería Química de la Universidad Central de Venezuela.

Da Fonseca-Rodríguez Andreina. Es ingeniera química por la Universidad Central de Venezuela, 2014. Actualmente es profesional de libre ejercicio en Venezuela.

Ruiz-Hernández Ernesto. Es ingeniero químico por la Universidad Central de Venezuela, 2014. Es profesional de libre ejercicio en Venezuela.

Pérez-Santodomingo Narciso. Es doctor en ingeniería por la Universidad Simón Bolívar, 2012, y MSc. en ingeniería química por la misma universidad, 2004. Es ingeniero químico por la Universidad Central de Venezuela, 2001 y profesor asociado adscrito al Departamento de Procesos y Sistemas, Universidad Simón Bolívar, Venezuela.

Rincón-Polo Gladys. Becaria Ateneo-Senescyt en la Facultad de Ingeniería Marítima, Ciencias Biológicas, Oceánicas y Recursos Naturales de la Escuela Superior Politécnica del Litoral-FIMCBOR-ESPOL, Guayaquil, Ecuador (2015). Es doctora en ingeniería de proyectos en medio ambiente por la Universidad Politécnica de Cataluña (2010), MSc. en investigación de operaciones (1986) e ingeniero químico por la Universidad Central de Venezuela (1983). Es profesor titular adscrito al Departamento de Procesos y Sistemas de la Universidad Simón Bolívar, Venezuela. 